\title{
ASO Visual Abstract: Functional Cerebral MRI Evaluation of the Integration of Breast Reconstruction into the Body Schema
}

Claudia Régis, $\mathrm{MD}^{1}$ (D), Marie-Cécile Le Deley, $\mathrm{PhD}^{2,3}$, Emilie Bogart, $\mathrm{MSc}^{2}$, Clémence Leguillette, $\mathrm{MSc}^{2}$, Loic Boulanger, $\mathrm{MD}^{1}$, Marie- Pierre Chauvet, $\mathrm{MD}^{1}$, Romain Viard, $\mathbf{P h D}^{4,5}$, Julien Thery, $\mathbf{M S c}^{6}$, Romain Bosc, $\mathrm{MD}, \mathrm{PhD}^{7}$, and Christine Delmaire, $\mathrm{MD}^{8}$

${ }^{1}$ Department of Surgery, Centre Oscar Lambret, Lille cedex, France; ${ }^{2}$ Biostatisitcs Unit, Centre Oscar Lambret, Lille cedex, France; ${ }^{3}$ Université Paris-Saclay, Universite' Paris-Sud, UVSQ, CESP, INSERM, Villejuif, France; ${ }^{4}$ University in Lille, CNRS, Inserm, CHU Lille, Institut Pasteur de Lille, US 41 - UMS 2014 - PLBS, Lille, France; ${ }^{5}$ University in Lille, Inserm, CHU Lille, U1172 - LilNCog - Lille Neuroscience \& Cognition, Lille, France; ${ }^{6}$ Clinical Research and Innovation Department, Centre Oscar Lambret, Lille, France; ${ }^{7}$ Department of Plastic, Reconstructive, Aesthetic and maxillofacial Surgery, Henry Mondor Hospital, Créteil, France; ${ }^{8}$ Imagering Department of Adolphe Rotschild Fondation, Paris, France

This prospective experimental study (https://doi.org/10. 1245/s10434-021-11048-0) sought to investigate the integration of reconstructed breast into the body schema, using functional magnetic resonance imaging. Findings suggested self-attribution of breast reconstruction, regardless of the type or the timing.

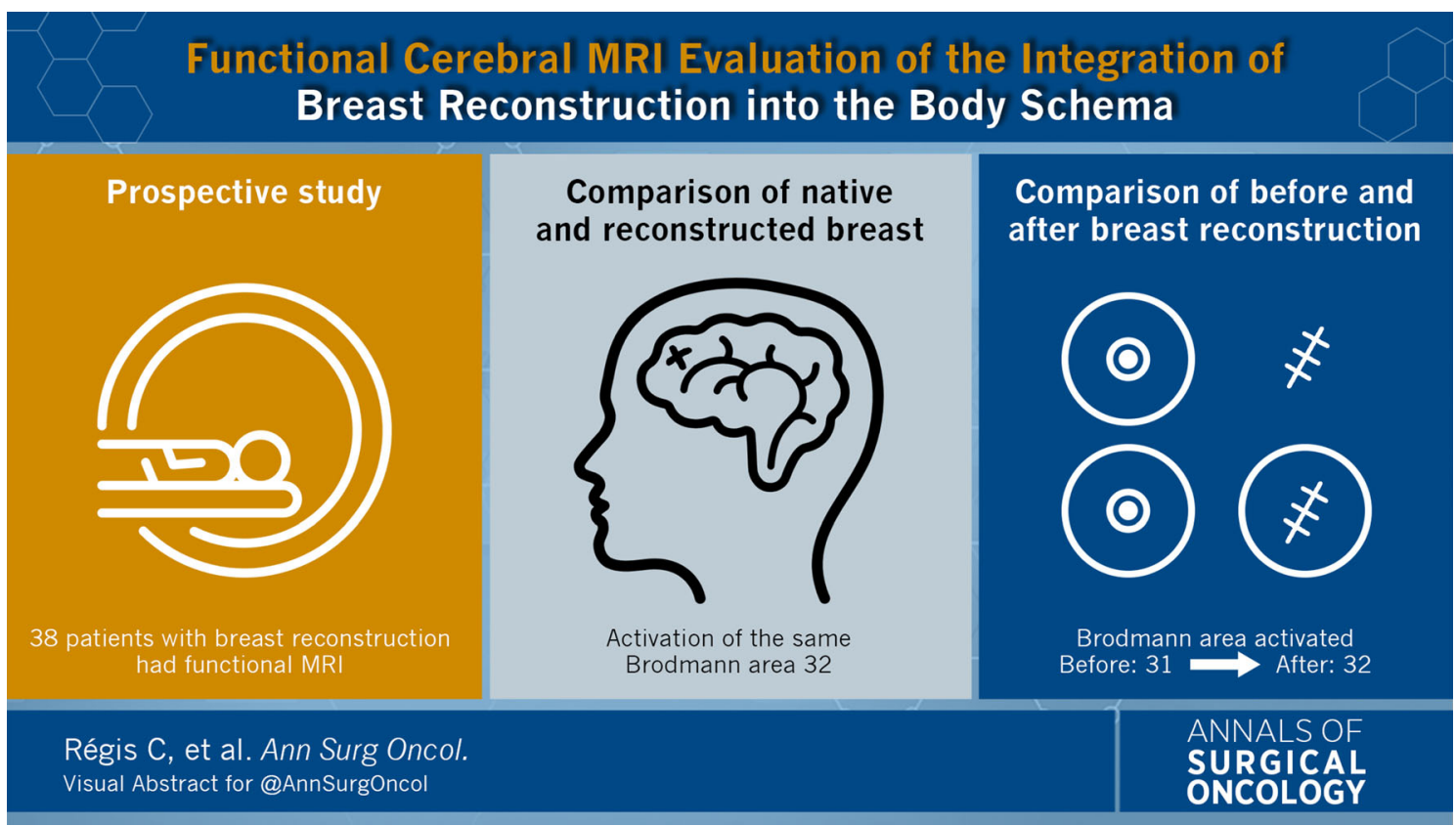

DISCLOSURE There are no conflict of interest.

(C) Society of Surgical Oncology 2021

C. Régis, MD

e-mail: c-regis@o-lambret.fr
Publisher's Note Springer Nature remains neutral with regard to jurisdictional claims in published maps and institutional affiliations. 